\title{
Nucleotide sequences and comparison of two large conjugative plasmids from different Campylobacter species

\begin{abstract}
Jerry M. Wells
Jerry.Wells@bbsrc.ac.uk
\end{abstract} \\ Correspondence \\ Received 23 February 2004 \\ Revised 9 June 2004 \\ Accepted 30 June 2004

\author{
Roger A. Batchelor, ${ }^{1}$ Bruce M. Pearson, ${ }^{2}$ Lorna M. Friis, ${ }^{2}$ Patricia Guerry ${ }^{1}$ \\ and Jerry M. Wells ${ }^{2}$ \\ ${ }^{1}$ Naval Medical Research Center, Silver Spring, MD 20910, USA \\ ${ }^{2}$ BBSRC Institute of Food Research, Norwich Laboratory, Norwich Research Park, Colney \\ Lane, Colney, Norwich NR4 7UA, UK
} \\ Two large tetracycline resistance $\left(\mathrm{Tc}^{\mathrm{R}}\right)$ plasmids have been completely sequenced, the pTet plasmid (45.2 kb) from Campylobacter jejuni strain 81-176 and a plasmid pCC31 $(44.7 \mathrm{~kb})$ from Campylobacter coli strain CC31 that was isolated from a human case of severe gastroenteritis in the UK. Both plasmids are mosaic in structure, having homologues of genes found in a variety of different commensal and pathogenic bacteria, but nevertheless, showed striking similarities in DNA sequence and overall gene organization. Several predicted proteins encoded by genes involved in conjugation showed highest homology to proteins found in Actinobacillus actinomycetemcomitans, a periodontal pathogen. In addition to replication- and conjugation-associated genes, both plasmids carried a tet(O) gene encoding tetracycline resistance, a $6 \mathrm{~kb}$ ORF encoding a putative methylase and a number of genes of unknown function. The pTet plasmid co-exists in C. jejuni strain 81-176 with a smaller, previously characterized, non-conjugative plasmid pVir that also encodes a type IV secretion system (T4SS) that may affect virulence. In contrast, the T4SS encoded by pTet and pCC31 are shown to mediate bacterial conjugation between Campylobacter. The possible origin and evolution of pCC31 and pTet is discussed.
}

\section{INTRODUCTION}

Campylobacter spp. account for the majority of bacteriarelated foodborne illness, with poultry and poultry products being reported as the major sources of infection in developed countries (Oberhelman \& Taylor, 2000). Approximately $80 \%$ of Campylobacter infections are caused by two species of the genus, namely, Campylobacter jejuni and Campylobacter coli, with the former being more frequently associated with disease in humans. Symptoms from infection with $C$. jejuni can vary from very mild diarrhoea to profuse bloody diarrhoea with mucosal damage and inflammation, especially in the ileum and jejunum (Wassenaar \& Blaser, 1999). In a rare number of cases infection with Campylobacter is associated with the peripheral neuropathies known as Guillain-Barré and Miller Fisher syndromes (Nachamkin et al., 1998).

Plasmids have played a major role in the ability of bacteria

Abbreviations: $T c^{R}$, tetracycline resistance; T4SS, type IV secretion system.

The sequence of the pTet and pCC31 plasmids have been deposited in GenBank under accession numbers AY394561 and AY394560, respectively. to exploit new environments, particularly under selective pressure, and are frequently associated with virulence attributes in pathogenic bacteria. Knowledge of plasmid genetics and the potential for conjugal transfer is therefore important for understanding the evolution and origin of transferable factors such as drug resistance genes. A survey of 688 human isolates of C. jejuni and C. coli in the USA revealed that $32 \%$ of strains harboured plasmid DNA, estimated to range in size from 2 to $162 \mathrm{~kb}$ (Tenover et al., 1985). A survey of 167 poultry samples and 41 clinical isolates of Campylobacter in Taiwan revealed a high occurrence of plasmids, 91 and $44 \%$, respectively (Lee et al., 1994). Of the tetracycline resistant strains surveyed, $87 \%$ of the chicken isolates and $47 \%$ of the clinical isolates carried the tet $(\mathrm{O})$ gene conferring tetracycline resistance $\left(\mathrm{Tc}^{\mathrm{R}}\right)$ on plasmids (Taylor, 1986; Taylor et al., 1981, 1986; Tenover et al., 1985). This high proportion of $\mathrm{Tc}^{\mathrm{R}}$ strains may reflect the farm use of tetracycline.

The well-characterized C. jejuni strain 81-176, originally isolated from a diarrhoeal outbreak associated with the consumption of unpasteurized milk (Korlath et al., 1985), contains two large $(>37 \mathrm{~kb})$ plasmids, pVir and a $\mathrm{Tc}^{\mathrm{R}}$ plasmid designated pTet (Bacon et al., 2000). Strain 81-176 has been shown to cause inflammatory diarrhoea in two 
human feeding studies as well as disease symptoms in experimental infection models using primates and ferrets (Black et al., 1988) (D. Tribble, unpublished, cited by Bacon et al., 2002). The DNA sequence of the non-conjugative plasmid pVir (37468 bp) was recently reported (Bacon et al., 2002). This plasmid has several genes that encode orthologues of type IV secretion systems (T4SS) and show their highest level of homology to a recently described T4SS of unknown function found in Helicobacter pylori $\mathrm{J99}$ (Kersulyte et al., 2003). T4SS have been reported in numerous pathogenic bacteria and play diverse roles including DNA export, bacterial conjugation and protein secretion [for review see Cao \& Saier, (2001)]. The precise role of the T4SS carried on pVir is unknown, although mutation of several pVir genes, including some but not all, T4SS homologues, resulted in reductions of invasion into INT407 cells in vitro and, for the one mutant that was tested, a reduction in virulence in the ferret diarrhoea model (Bacon et al., 2000, 2002). Additionally, mutation of a subset of $\mathrm{pVir}$ genes affected natural transformation (Bacon et al., 2000).

In order to gain further insight into the structure and function of Campylobacter plasmids we have completely sequenced two large $\mathrm{Tc}^{\mathrm{R}}$ plasmids, the pTet plasmid $(45 \cdot 2 \mathrm{~kb})$ from C. jejuni strain $81-176$ and a plasmid pCC31 $(44 \cdot 7 \mathrm{~kb})$ from C. coli strain CC31 that was isolated from a human case of severe gastroenteritis in the UK. Strikingly, these two plasmid sequences revealed a remarkable level of sequence identity despite the fact that the strains were isolated almost 20 years apart on different continents. Sequence analysis of the two plasmids revealed genes encoding a putative T4SS that has been shown to be involved in conjugation, and is distinct from the T4SS system found on C. jejuni virulence plasmid pVir. Both $T c^{\mathrm{R}}$ plasmids also encode a number of genes whose proteins best match those found in $H$. pylori, including one gene from the plasticity zone of $H$. pylori $J 99$ (Alm et al., 1999).

\section{METHODS}

Bacterial strains and plasmids. C. jejuni and C. coli strains used this study are shown in Table 1. Campylobacter were grown on Mueller-Hinton (MH) medium at 42 or $37^{\circ} \mathrm{C}$ under microaerobic conditions in the presence of the following antibiotics, when appropriate: $20 \mu \mathrm{g}$ tetracycline $\mathrm{ml}^{-1}, 20 \mu \mathrm{g}$ streptomycin $\mathrm{ml}^{-1}, 20 \mu \mathrm{g}$ kanamycin $\mathrm{ml}^{-1}$, and/or $20 \mu \mathrm{g}$ chloramphenicol $\mathrm{ml}^{-1}$. Plasmids pUC19 and pBluescript were used as the cloning vectors and Escherichia coli $\mathrm{DH} 5 \alpha$ was the host for cloning experiments. E. coli strains were grown at $37^{\circ} \mathrm{C}$ on Luria-Bertani (LB) broth supplemented with $50 \mu \mathrm{g}$ ampicillin $\mathrm{ml}^{-1}$ or $20 \mu \mathrm{g}$ chloramphenicol ml $\mathrm{m}^{-1}$ as required.

DNA recombinant techniques. Plasmid DNAs were isolated using mini-Qiagen columns as previously described by Bacon et al., (2000). Restriction enzymes were purchased from New England Biolabs and used as recommended by the supplier. Plasmid DNA samples for sequence analyses were isolated using QIAprep spin miniprep columns (Qiagen). DNA sequencing was performed using Big Dye sequencing kits (Perkin Elmer-Applied Biosystems) on Applied Biosystems 373A and 3100 DNA sequencers.

Sequencing of the pCC31 plasmid. A basic shotgun approach was taken to sequence the plasmid isolated from C. coli strain CC31. Plasmid DNAs digested with different restriction enzymes (Sau3A, HpaII and TaqI) were ligated into pUC19 multiple cloning sites and transformed into E. coli. Colonies were selected on LB agar containing $100 \mu \mathrm{g}$ ampicillin $\mathrm{ml}^{-1}$ and $0.5 \mathrm{mg} \mathrm{X}-\mathrm{Gal}_{\text {plate }}{ }^{-1}$ to identify white colonies containing vectors with recombinant DNA inserts. These clones were then sequenced using standard universal forward and reverse primers. Following this initial phase, the physical gaps and sequence gaps were closed by 'primer-walking' using a series of 20 mer primers designed on the sequence of the contigs obtained by shotgun sequencing. In total, 829 sequence reads were used to sequence the plasmid pCC31. Sequences were assembled using Seqman 5.05 software.

Sequencing of the pTet plasmid. Total plasmid DNA from 81-176 (comprising pTet and pVir) was digested with BglII, and pTet-specific fragments were purified from agarose gels (Bacon et al., 2000) and cloned into pBluescript (Stratagene). Clones were sequenced by a combination of primer-walking, using synthetic oligonucleotide primers, and by an in vitro transposition strategy using a previously described EZ:: TN system (Epicentre) containing a Campylobacter chloramphenicol resistance gene (Yao et al., 1993), as previously described by Guerry et al. (2000). Sequence gaps were closed by using primers based on the sequences at the end of the contigs to PCR amplify linking DNA fragments. Sequences were assembled using Sequencher 4.1 software.

Synthetic oligonucleotides. Synthetic oligonucleotides for DNA sequencing and PCR were either synthesized on an Applied Biosystems model 393 DNA synthesizer or purchased from Sigma Genosys.

\section{Table 1. Strain list}

$\mathrm{Nal}^{\mathrm{R}}$, Nalidixic acid resistant; $\mathrm{Str}^{\mathrm{R}}$, streptomycin resistant; $\mathrm{Tc}^{\mathrm{R}}$, tetracycline resistant; cat, chloramphenicol acetyltransferase; aph3, kanamycin resistance.

\begin{tabular}{|c|c|c|}
\hline Strain & Description & Source or Reference \\
\hline C. jejuni 81-176 & WT: pTet $\left(\mathrm{Tc}^{\mathrm{R}}\right)$ pVir & Korlath et al. (1985) \\
\hline C. jejuni 1769 & 81-176: $\mathrm{pTet} / \mathrm{cmgB} 3 / 4::$ cat $\left(\mathrm{Tc}^{\mathrm{R}}\right) \mathrm{pVir}$ & This work \\
\hline C. jejuni DB179 & 81-176: pVir/cjp8:: cat & Bacon et al. (2000) \\
\hline C. jejuni 847 & 81-176: recA::aph3 pVir & Guerry et al. (1994) \\
\hline C. jejuni VC83 & Plasmid-free, $\mathrm{Nal}^{\mathrm{R}}, \mathrm{Str}^{\mathrm{R}}$ & Guerry et al. (1994) \\
\hline C. jejuni 2048 & VC83: pTet $\left(\mathrm{Tc}^{\mathrm{R}}\right)$ & This work \\
\hline C. coli CC31 & WT: pCC31 $\left(\mathrm{Tc}^{\mathrm{R}}\right)$ & D. Ala'Aldeen, Nottingham \\
\hline
\end{tabular}


Annotation. The finished plasmid sequences were oriented starting at the first base of the tet $(\mathrm{O})$ gene, and annotated manually. ORFs of greater than 50 residues were evaluated based on the presence of a suitable initiation codon with appropriate spacing to a ribosomebinding site as well as physical location to other ORFs.

Full-length nucleotide and polypeptide sequences of all plasmidencoded ORFs greater than 50 amino acids in length were searched for matches against all available public sequence databases using the BLAST algorithm (http://www.ncbi.nlm.nih.gov/blast/). Levels of identity and homology were calculated across the full length of the plasmid proteins by alignment of sequences in DNAMAN (Lynnon Corporation). Additionally selected polypeptide homologues were aligned and compared using CLUSTALX. Prosite (http://ca.expasy.org/prosite/) was used to identify conserved functional motifs in protein sequences. Putative promoter regions were identified using the Neural Network Promoter Prediction program (http://www.fruitfly.org/seq_tools/promoter. html). Sequences were identified based on a cut-off score of 0.8 and their location with respect to the ribosome-binding site.

Mutagenesis of pTet. A non-polar insertion of the CAT transposon into the $c m g B 3 / 4$ gene, used for DNA sequence analysis, was selected; this particular transposon insertion mapped to $1700 \mathrm{bp}$ within the coding region of $\mathrm{cmgB3/4}$. The clone was used to electroporate $81-176$ to chloramphenicol resistance $\left(\mathrm{Cm}^{\mathrm{R}}\right)$ as previously described by Bacon et al. (2000). Putative mutants were analysed by PCR using primers flanking the insertion point to confirm that a double crossover event had occurred.

Conjugations. Conjugations between Campylobacter strains were performed by a modification of the methods previously described by Kuipers et al. (1998) and Taylor et al. (1981). Campylobacter strains listed in Table 1 that were used as donor stains in mating experiments were made recA-negative by crossing them with a $C$. jejuni strain that had a kanamycin-resistant cassette (aph3) inserted into the $r e c A$ gene (Guerry et al., 1994). Briefly, strains were grown overnight on selective $\mathrm{MH}$ plates, harvested in $1 \mathrm{ml} \mathrm{MH}$ broth at a density of approximately $10^{9}$ c.f.u. $\mathrm{ml}^{-1}$ and combined in $100 \mu \mathrm{l}$ aliquots on $\mathrm{MH}$ plates without antibiotics. DNaseI (Roche) was added to the suspension at a final concentration of $10 \mathrm{U} \mathrm{\mu l}^{-1}$ to prevent transfer of plasmid by natural transformation and/or transfer of counter-selectable markers from the intended recipient into the intended donor. After incubation for $12-16 \mathrm{~h}$ at $37^{\circ} \mathrm{C}$ under microaerobic conditions, bacteria were removed with a sterile swab, dispersed in $1 \mathrm{ml}$ of $\mathrm{MH}$ broth and plated at different dilutions on $\mathrm{MH}$ containing the appropriate antibiotics. Transconjugants obtained were tested for the flagellin polymorphisms (Alm et al., 1993) that distinguished donor and recipient strains to confirm conjugal transfer (data not shown).

\section{RESULTS}

\section{General description of pCC31 and pTet}

A physical map comparing the two plasmids maps is shown in Fig. 1 and gene annotations are presented in Table 2. The sequence of pCC 31 was determined to be $44707 \mathrm{bp}$ and annotation revealed 50 ORFs, 44 of which are transcribed in a clockwise orientation with respect to the $\operatorname{tet}(\mathrm{O})$ gene. The sequence of pTet was determined to be $45205 \mathrm{bp}$, only $1 \%$ larger than pCC 31 . Annotation of the sequence revealed 49 ORFs, 43 being transcribed in a clockwise orientation. The two plasmids are $94.3 \%$ identical at the level of nucleotide sequence. Approximately $90.0 \%$ of both plasmids is coding sequence, with overall $\mathrm{G}+\mathrm{C}$ contents of $29 \cdot 8 \%$ (pCC31) and $29 \cdot 1 \%$ (pTet), which are only slightly lower than that of the sequenced $C$. jejuni genome (30.6\%) (Parkhill et al., 2000). However, the $\mathrm{G}+\mathrm{C}$ plot revealed a high $\mathrm{G}+\mathrm{C}$ region incorporating the $\operatorname{tet}(\mathrm{O})$ gene $(40 \cdot 4 \% \mathrm{G}+\mathrm{C})$ suggesting that this gene was horizontally transferred from another species (Fig. 1c).

Conjugative plasmids encoding tet $(\mathrm{O})$ from Campylobacter have been described in detail by Taylor et al. (1981) although only the sequence of the Tet $\mathrm{O}$ determinant and limited adjacent DNA from pUA466 has been reported (Taylor, 1986). The $45 \mathrm{~kb}$ pUA466 plasmid was subsequently found to have a different restriction pattern to the plasmids described in this manuscript (data not shown) (Taylor et al., 1986). The tet(O) gene shares significant homology with the tet $(\mathrm{M})$ gene of Streptococcus and is thought to have a shared ancestry. The upstream promoter regions of tet $(\mathrm{O})$ on both pTet and pCC31 are highly conserved with the published sequences (Taylor, 1986). The two plasmid tet $(\mathrm{O})$ genes are $94.8 \%$ homologous at the DNA level with almost all differences occurring within a central $350 \mathrm{bp}$ region.

A cluster of five 15 bp direct repeats (ATTACATTTAAGTCA) was found in the intergenic region between $c p p 23$ and cpp24 (bp 20889-21160), as indicated by the open box in Fig. 1. Such repetitive regions are characteristic of replication origins (Konieczny, 2003) suggesting that these sites may function as such in these $\mathrm{Tc}^{\mathrm{R}}$ plasmids.

\section{Differences between the two plasmids}

The major difference between pTet and pCC31 occurs within a region of bp 14333-18803 of pTet and bp 14022-18699 of pCC31. Plasmid pCC31 contains a gene (cpp15) at bp 14159-14887 encoding a protein with $45 \%$ identity and $64 \%$ similarity to a hypothetical protein from H. pylori 26695 (Tomb et al., 1997) (see below). Plasmid pTet lacks cpp15 but has an additional ORF (cpp21) at bp 18150-18803 that encodes a protein with $33 \%$ identity and $50 \%$ similarity to JHP1408, a hypothetical protein from H. pylori 599 (Alm et al., 1999). Additional H. pylori alleles are found on both plasmids (see below). Additionally, there is a small ORF designated gene $c p p 48$ on pCC31 (but not pTet) that encodes a predicted protein of $6 \mathrm{kDa}$ that shows no significant homology to known proteins.

The majority of matching genes contain small numbers of base substitutions generally giving rise to polypeptides which are predicted to be identical in length. In addition there are 19 cases where the alleles have different predicted lengths, e.g. in pCC31, $c p p 46$ starts with ATGATG whereas in pTet this gene starts with only one ATG; in $s s b 1, c m g B 7$ and $c m g B 8$ an additional $3 \mathrm{bp}$ is present at the end of the gene in pTet. Similarly, genes $c m g B 9, c m g B 10$ and cpp44 have 3 or 6 bp additions at different locations in each allele. Some genes have modified 3 ' ends where the reading frames and stop codon of one allele appear to have been shifted by addition or deletion of bases (e.g. $c p p 2,11$ bp; $c p p 16$, $7 \mathrm{bp} ; \mathrm{cmgB3} / 4,1 \mathrm{bp}$ ). In addition, the ORF of some genes 
(a)

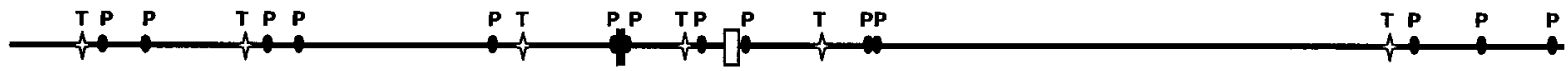

(b)

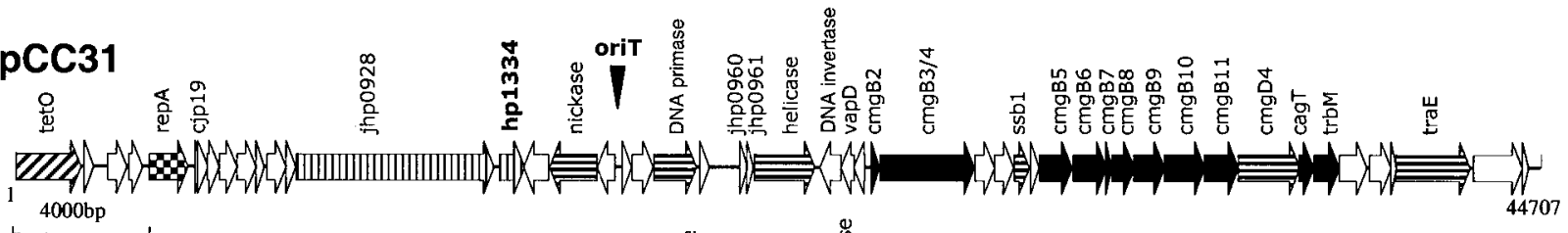

pTet

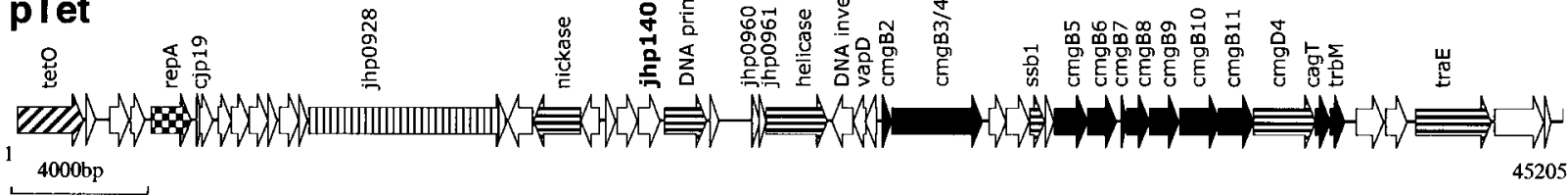

(c) pTet G + C Content (\%)

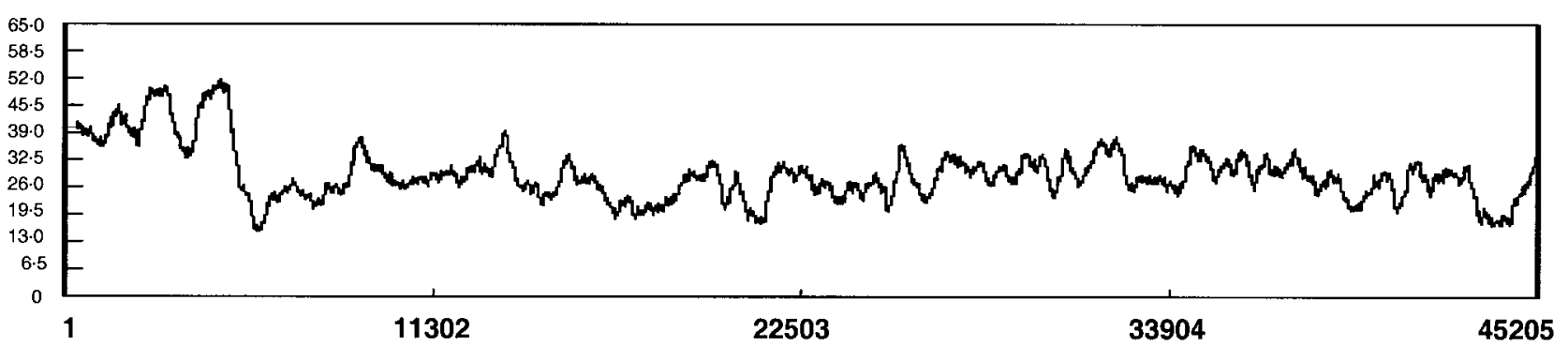

Fig. 1. Genetic map of plasmids pTet and pCC31 showing differences in gene organization. (a) Putative promoters (designated $\mathrm{P}$ ) and transcription terminators (designated T) identified in pCC31 are indicated, as is the inverted repeat region (filled box) containing the proposed nic site and origin of transfer (oriT). A region of multiple direct repeats is also indicated by an open box. (b) Genes are annotated according to Table 2 and identified by their predicted function as follows: DNA transfer functions, horizontal stripes; Campylobacter mating gene $(\mathrm{cmg})$ homologues of T4SS, filled; repA, chequered; restriction modification, vertical stripes; tet(O), diagonal stripes; genes with unknown role, open. Genes that are only found in one of the two plasmids are labelled in bold. (c) $G+C$ content. Due to the similarity of $G+C$ content between the two plasmid sequences only that of pTet is displayed.

is shifted near to the start, including $c p p 32$ which has several other point mutations over the entire length, and cpp33 which varies considerably between pCC31 and pTet. As the function of these genes is unknown, we cannot predict which of the frame-shifted variants is a pseudogene. The $v a p D$ homologue in pCC31 has an additional 30 bp near the $3^{\prime}$ end, and in pTet $c p p 7$ and $c p p 8$ are both affected by a 299 bp section of additional DNA.

\section{Genes encoding putative maintenance functions}

A putative replication initiator protein, RepA was identified on the basis of its similarity to the rep protein on pTS1 from Treponema denticola. Similar to the broad-host-range plasmid pIPO2, this putative repA gene is embedded in putative ORFs of unknown function (Tauch et al., 2002).

\section{Genes encoding putative conjugation and T4SS homologues}

There are 10 genes in pTet and pCC31 that encode predicted proteins with homology to T4SS proteins, in a region spanning approximately $12 \cdot 6 \mathrm{~kb}$. T4SS are multicomponent complexes spanning the cell envelope that can translocate proteins and/or nucleoprotein complexes between bacteria (Cao \& Saier, 2001). Corresponding systems found on some plasmids of Gram-negative bacteria are responsible for mating pair formation (Mpf), involving pilus assembly and initial contact to the recipient cell during conjugation and DNA transfer (Christie \& Vogel, 2000). Most of the T4SS components encoded by pCC31 and pTet show their highest homologies to proteins involved in conjugation of plasmids in Gram-negative bacteria, primarily to the pVT745 plasmid from Actinobacillus actinomycetemcomitans, a periodontal pathogen (Galli et al., 2001). Accordingly, these plasmid genes with homology to the T4SS have been designated $\mathrm{cmg}$ (Campylobacter mating genes) to indicate their putative role in the formation of a transfer apparatus (see below) and are numbered according to their functional homologues in the archetypal vir transfer system of Agrobacterium tumefaciens (Table 2, Fig. 1). Agrobacterial TDNA transfer systems typically comprise between 10 and 15 genes in a single cluster, which encode the membrane 
Table 2. Predicted coding regions on pTet and pCC31 plasmids and the closest relationships to previously studied proteins

A. denitrificans, Achromobacter denitrificans; B. cepacia, Burkholderia cepacia; C. jejuni, Campylobacter jejuni; C. crescentus, Caulobacter crescentus; C. hutchinsonii, Cytophaga hutchinsonii; E. faecalis, Enterococcus faecalis; G. sulfurreducens, Geobacter sulfurreducens; H. influenzae, Haemophilus influenzae; R. anatipestifer, Riemerella anatipestifer; S. typhimurium, Salmonella typhimurium; S. oneidensis, Shewanella oneidensis; T. denticola, Treponema denticola; W. succinogenes, Wolinella succinogenes; Y. pestis, Yersinia pestis. For genes cpp32, ssb, cmgB8, cmgB9, cmgB10 and $c m g B 11$, the highest homology hits were to C. jejuni genes/proteins with GenBank accession numbers AY190288, NC_005012, AY190284, AY190285, AY190286 and AY190287, respectively.

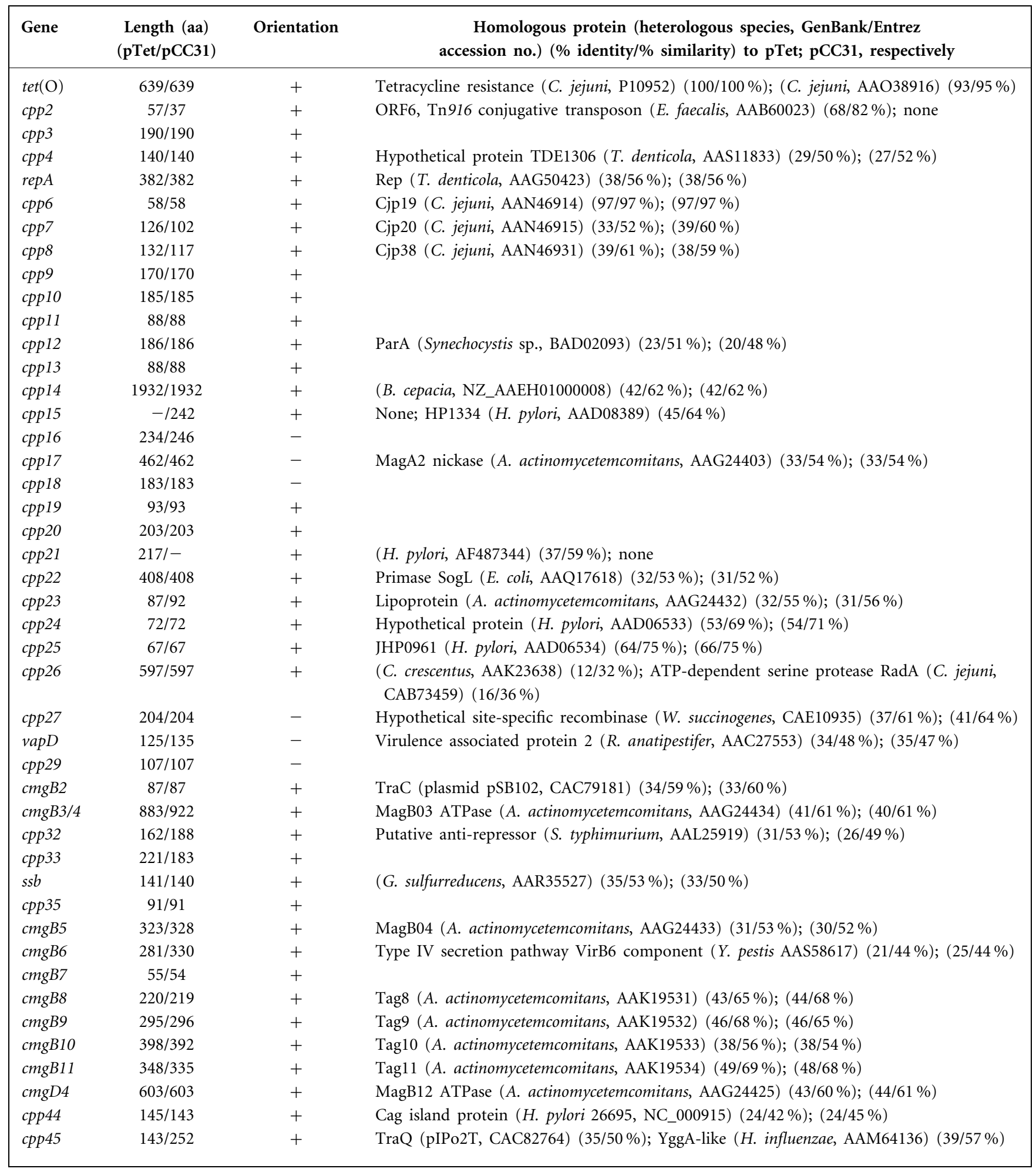


Table 2. cont.

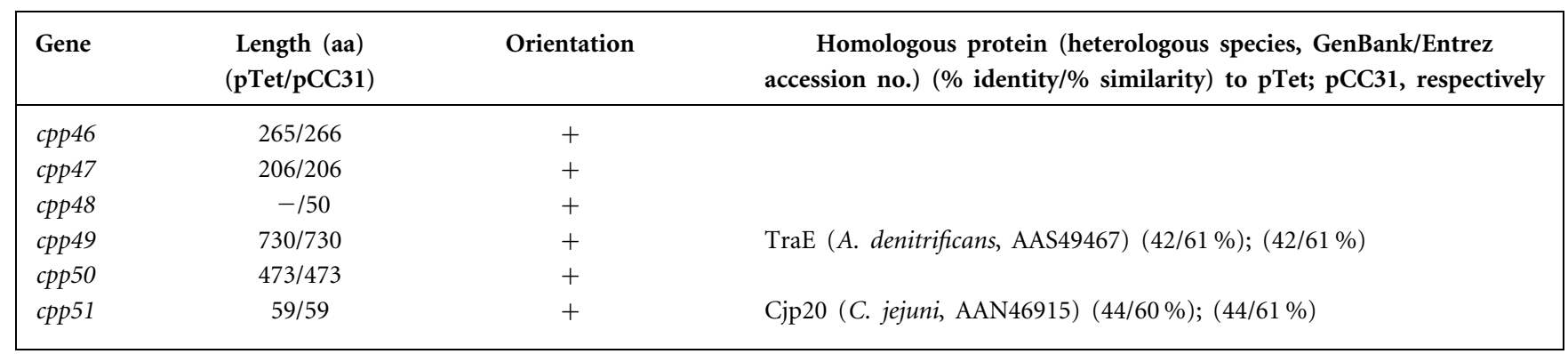

pilus (VirB2), a trans-envelope pore complex (VirB6-10), a transfer coupling protein (VirD4) and cytoplasmic membrane ATPases (VirB4 and VirB11; Fig. 1). Similarly, the $\mathrm{cmg}$ genes in pCC31 and pTet are organized in what is predicted to be a single transcription unit (Fig. 1). The location of $\mathrm{cmgD4}$ (a homologue of virD4) (Balzer et al., 1994; Lessl et al., 1992; Moncalian et al., 1999), cpp44 (a homologue of cagT) and cpp45 trbM at the end of the cmg operon is, however, unusual but is also found in the conjugative plasmid pVT745 from Actinobacillus actinomycetemcomitans (Galli et al., 2001). The trbMgene has only been found in the IncP-specific transfer operon and its role in conjugation, if any, is unknown (Pansegrau \& Lanka, 1996).

Both $\mathrm{Tc}^{\mathrm{R}}$ plasmids encode a VirB2 or pilin homologue ( $c m g B 2)$; this represents the first pilin gene identified in Campylobacter (Gaynor et al., 2001; Parkhill et al., 2000). The highest homology of this predicted protein is to TraC from plasmid pIP02T, a broad-host plasmid found in a variety of plant rhizosphere bacterial symbionts (Tauch et al., 2002). Like other pilins these Campylobacter plasmidencoded VirB2 proteins contain putative signal peptides, predicted to be cleaved between amino acid position 18 and 19 to generate a small basic protein of $9 \mathrm{kDa}$. Generally, the signal peptides of VirB2 preproteins are longer (25-50 amino acids long). Electron microscopic examination of our strains did not reveal evidence of pili, as previously reported for 81-176 (Gaynor et al., 2001). Alignment of the C-terminal region of the $\mathrm{cmgB2}$ pilin found in pTet and pCC31 with other pilins revealed that the four amino acid residues removed by the TraF protease during the cyclization of other pilins were completely conserved (Eisenbrandt et al., 2000). However, no obvious homologue of TraF was found in pTet or pCC31. A homologue of the VirB2-associated gene VirB5 (cmgB5) is present in both pCC31 and pTet. VirB5 is reported to be a minor component of the agrobacterial T-pilus (Table 2) (SchmidtEisenlohr et al., 1999). CmgB5 also shows its strongest homology to the VirB5 homologue from Actinobacillus actinomycetemcomitans (31\% identity and 54\% similarity).

Both plasmids encode homologues of VirB6, B7, B8, B9 and B10 proteins from Actinobacillus actinomycetemcomitans, as shown in Table 2. CmgB6, the VirB6 homologue, is predicted to form five transmembrane helices and thus might form a channel in the cytoplasmic membrane. Both plasmids contain a putative, small, 54-55 amino acid protein encoded by $\mathrm{cmgB7}$. CmgB7 has no homology to other proteins by BLASTP analysis because of its small size, but like the small VirB7 protein of Agrobacterium and the MagB07 protein from the mating gene operon of pVT745, it contains a lipoprotein signal sequence and conserved lipid attachment site, suggesting that these genes might have a similar function (Galli et al., 2001). In Agrobacterium VirB7 has been shown to form disulphide bonds with VirB9 and stabilize the other VirB proteins during T-pilus assembly (Anderson et al., 1996; Spudich et al., 1996). It is also possible that this protein plays a role in entry exclusion, as found for the small lipoprotein designated TrbK in the conjugative IncP (RP4) plasmid transfer system (Pansegrau \& Lanka, 1996). Like CmgB7 and the entry exclusion protein of the E. coli $\mathrm{F}$ plasmid, TrbK has a lipoprotein signal sequence at its $\mathrm{N}$ terminus, suggesting that it is exposed at the cell surface. TrbK mutants of RP4 lack a pilus suggesting that TrbK interacts with Mpf apparatus, although this is not essential for conjugative DNA transfer (Vergunst et al., 2000). Interestingly, both plasmids encode a second allele of VirB7 encoded by cpp44. Cpp44 shows its highest homology to CagT, the VirB7 homologue encoded by the Cag pathogenicity island of H. pylori (Censini et al., 1996).

The $c m g B 9$ gene found in both $\mathrm{Tc}^{\mathrm{R}}$ plasmids shares its highest homology with a VirB9-like protein identified in another C. jejuni plasmid (R. Schmidt-Ott, University of Göttingen, Germany, unpublished data; GenBank AY190285), and contains a putative signal peptide suggesting that it might be transported into the periplasm where it can interact with other components of the membrane spanning complex. Interestingly, the pTet- and pCC31encoded proteins with homology to VirB8 and VirB10 both contain single transmembrane helices near the $\mathrm{N}$ terminus. This suggests that the proteins orientate such that a short $\mathrm{N}$-terminal domain remains in the cytoplasm and a larger C-terminal domain is located in the periplasm. The VirB10 protein of Agrobacterium has the same predicted topological feature and the carboxy-terminal periplasmic domain is thus proposed to link the cytoplasmic and outer-membrane proteins of the mating pair channel (Beaupre et al., 1997).

Both plasmids encode homologues of the three ATPases associated with T4SS, namely VirB11 (CmgB11), VirD4 
(CmgD4; a transfer coupling protein) and VirB4 (CmgB3/4; a probable lipoprotein). All three of these predicted proteins show high homology to genes from Actinobacillus actinomycetemcomitans. Like other homologues of these ATPases the Campylobacter proteins contain Walker A nucleotidebinding motifs and the conserved motifs B-D that were previously shown to be essential for conjugation and phage absorption in E. coli (Krause et al., 2000; Schmidt-Eisenlohr et al., 1999). The VirB11 homologues in pTet and pCC31 do not possess any obvious features associated with membraneor periplasmic-located proteins and thus might interact with the cytoplasmic domains of the other components of the $\operatorname{Vir} B$ protein channel complex, as previously suggested by Thorstenson et al. (1993).

\section{Genes encoding putative DNA transfer enzymes (Dtr)}

As on plasmid pSB102 (Schneiker et al., 2001), the putative genes for the processing of DNA for transfer (Dtr) and establishment of the plasmids in the recipient cell are scattered across both Campylobacter plasmids. Conjugative DNA transfer in the Enterobacteriaceae requires the formation of a nucleoprotein complex called the relaxosome (Cao \& Saier, 2001). Following cleavage by the nickase at the origin of DNA transfer (oriT), a strand replacement reaction generates a single-stranded DNA transfer intermediate (T-strand) that presumably moves with the attached proteins to dock with the DNA transfer apparatus. The Dtr processing enzymes that assemble to form the relaxosome determine the site specificity of cleavage and control the timing of DNA transfer so that it does not interfere with vegetative replication of the plasmid. The main feature of oriT is the presence of an inverted repeat adjacent to the specific cleavage site (called the 'nic' site) of the nickase/replicase (Pansegrau \& Lanka, 1996). The non-coding region between $c p p 18$ and $c p p 19$ in pTet and pCC31, designated 'oriT' in Fig. 1, may function as the oriT region since it contains inverted DNA repeats surrounding a conserved 'nic' site motif ATCCTG as found in other oriT sites (Fig. 2) (Pansegrau \& Lanka, 1996). Moreover, this site lies close to the DNA processing enzymes as found in other conjugative plasmids (Fig. 1). pVir is non-conjugative and no sequence homology was found to the oriT sites described here.

The pCC31 and pTet CmgD4 proteins share homology with the transfer coupling proteins VirD4 (Ti plasmid) and $\operatorname{TraG}$ (F plasmid) that are required for recruiting the relaxosome nucleoprotein complex and coupling it to the Mpf DNA transfer apparatus in the cell envelope (Zechner et al., 2000). T4SS coupling proteins are required for DNA or protein transfer in Agrobacterium tumefaciens, H. pylori and bacterial conjugation systems (Cabezon et al., 1994; Covacci et al., 1999; Moncalian et al., 1999; Vergunst et al., 2000). However, some T4SS are devoted to export of proteins such as the Bordetella pertussis toxin and so lack the transfer coupling proteins.

\begin{tabular}{ll} 
& \multicolumn{1}{c}{ 'nic' site } \\
pCC31 & TTTGAGAAATAAAAGGCTATCCTGCAATCATTAAATTATTTC \\
pTet & TTTGAGAAATAAAAGGCTATCCTGCAATTATCAATTATTAAA \\
plP02 & TTTACCGGCGATTAGGCTATCCTGCAATAGCCCACACCCCCC \\
pSB102 & CTTTACCGGCGTTAGCCTATCCTGCAATAGACCTCACAAGCC \\
RP4 & GGTGGGCCTACTTCACCTATCCTGCCCGGCTGACGCCGTTGG \\
pXF51 & ATAGCGGTTTTAAAACCTATCCTGCCCTAGATTTAACCCTCT \\
pTiC58 & TTGTTTACACCACAATATATCCTGCCACCAGCCAGCCAACAG \\
pTiA6 & CCATTTACAATTGAATATATCCTGCCGCCGCTGCCGCTTTGC \\
R751 & GGTTAGCTAACTTCACACATCCTGCCCGCCTTACGGCGTTAA
\end{tabular}

Fig. 2. The proposed oriT sequence of pCC31 and pTet are aligned to the conserved nic regions of $\mathrm{IncP}$ and $\mathrm{Ti}$ plasmids (Zechner et al., 2000) as well as the putative oriT of plasmids pIPO2, pSB102 and pXF51. Nucleotide sequences that are completely conserved are underlined. Bold type indicates nucleotide positions that are at least $70 \%$ conserved among the aligned sequences. The arrow shows the position of the nic site determined for the IncP transfer system.

Both plasmids encode a putative DNA nickase (cpp17) and a helicase ( $c p p 26)$ involved in generating a single stand, both with closest similarity to homologues in plasmid pVT745 from Actinobacillus actinomycetemcomitans, and a single-stranded DNA-binding protein ssb1 that may coat the single-stranded DNA during transfer, as in the case of the VirE2 ssb in Agrobacterium tumefaciens (Christie et al., 1988). Cpp22 in both plasmids has significant homology to the SogL primase of E. coli plasmid R64 and possesses a functional variant of the EGYATA motif associated with the active site of other primases (Strack et al., 1992). The SogL primase is transferred along with the transferred plasmid DNA and is thought to catalyse the synthesis of short oligonucleotides on the single-stranded template that are then elongated by the recipient replication machinery (Pansegrau \& Lanka, 1996).

\section{Genes encoding homologues of $\boldsymbol{H}$. pylori proteins}

In addition to HP1334, JHP1408 and cagT, discussed above, there are three other homologues of $H$. pylori genes encoded by both pTet and pCC31. cpp14 encodes a large protein (predicted molecular mass $224 \mathrm{kDa}$ ) that shows $37 \%$ identity and $55 \%$ similarity to JHP0928, a protein encoded in the plasticity zone of $H$. pylori $J 99$. Plasticity zones are regions of hypervariable genes in the chromosomes of $H$. pylori strains (Alm \& Trust, 1999). Most of these plasticity zone genes appear to be $H$. pylori-specific, but several homologues have been found in C. jejuni 81176 on pVir (Bacon et al., 2000). Although not originally annotated as a methylase (Alm et al., 1999), JHP0928, like Cpp14, shows homology to a putative methylase encoded by Sinorhizobium meliloti phage PBC5 (GenBank accession no. NC_003324). Genes cpp24 and cpp25 encode homologues of JHP0960 (54\% identity, $70 \%$ similarity) and JHP0961 (70 \% identity, $80 \%$ similarity), respectively, both small proteins of unknown function from H. pylori J99. 
Table 3. Mating frequency of Campylobacter strains harbouring pTet or pCC31

Chl, Chloramphenicol; Str, streptomycin; Tet, tetracycline.

\begin{tabular}{|c|c|c|c|}
\hline Genotype & Recipient & Selection & Frequency \\
\hline 81-176 recA::aph3 (pVir, pTet) & VC83 $\operatorname{Str}^{\mathrm{R}}$ & Str, Tet & $10^{-5}-10^{-6}$ \\
\hline 81-176 recA::aph3 (pVir, pTet) & DB179 (pVir/cjp8::cat) & Chl, Tet & $10^{-4}$ \\
\hline 81-176 recA::aph3 (pVir, pTet/cmgD4::cat) & VC83 $\operatorname{Str}^{\mathrm{R}}$ & Str, Tet & 0 \\
\hline VC83 recA::aph3 $\operatorname{Str}^{\mathrm{R}}$ (pTet) & DB179 (pVir/cjp8::cat) & Chl, Tet & $10^{-4}$ \\
\hline CC31 recA::aph3 (pCC31) & VC83 $\operatorname{Str}^{\mathrm{R}}$ & Str, Tet & $10^{-4}-10^{-5}$ \\
\hline DB179 recA::aph3 (pCC31, pVir) & VC83 $\operatorname{Str}^{R}$ & Str, Tet & $10^{-5}-10^{-6}$ \\
\hline CC31 recA::aph3 (pCC31) & DB179 (pVir/cjp8::cat) & Chl, Tet & $10^{-4}$ \\
\hline
\end{tabular}

\section{Genes encoding other proteins of predicted function}

There is a cluster of three genes transcribed in the opposite direction to the $c m g$ operon ( $c p p 27, c p p 28$ and $c p p 29)$. $c p p 29$, which appears to be the first gene in this putative operon, encodes a predicted protein of $12 \mathrm{kDa}$ that shows no homology to known proteins. cpp28 encodes a predicted protein of $15-16 \mathrm{kDa}$ that shows significant homology (35\% identity, $47 \%$ similarity) to VapD2 of Rhodococcus equi, an important pulmonary pathogen of foals (Takai et al., 2000). The precise role of vapD2 and the other vap genes in virulence is not known, and the sequence does not reveal any other clues to their function. $c p p 27$ encodes a predicted protein of $24 \mathrm{kDa}$ that shows $33 \%$ identity and $56 \%$ similarity to an invertase from Shewanella oneidensis. Invertases and resolvases have been identified on a variety of bacterial plasmids of both Gram-negative and Grampositive origin and have been shown to play roles in plasmid (Janniere et al., 1993) and genomic replication (Alonso et al., 1995; Bruand et al., 1995).

\section{Conjugal transfer}

Although conjugative transfer of Tet $\mathrm{O}$ plasmids has been reported previously (Taylor et al., 1981), preliminary experiments (Bacon et al., 2000) to determine if 81-176 could conjugally transfer either pVir or pTet were inconclusive, in large part because of problems in distinguishing conjugation from natural transformation (Bacon et al., 2000). The ability of 81-176 to conjugally transfer pTet to several recipients was re-examined using a $\operatorname{rec} A:: a p h 3$ mutant as donor (Guerry et al., 1994), as shown in Table 3. A recA mutant of 81-176 was able to transfer the pTet plasmid to a recipient strain of $C$. jejuni (VC83) that lacked plasmids and contained a $\operatorname{Str}^{\mathrm{R}}$ chromosomal marker (Guerry et al., 1994), at a frequency of $10^{-5}-10^{-6}$ per donor cell. A derivative of 81-176 (DB179) lacking pTet (Bacon et al., 2000) and marked by insertion of a $\mathrm{Cm}^{\mathrm{R}}$ marker into Cjp8 of pVir (Bacon et al., 2002) was found to receive pTet from $81-176$ at a frequency of $10^{-4}$ per recipient cell. These observed differences in frequency of conjugation are suggestive of a restriction barrier in a heterologous VC83 recipient. Interestingly, the same transfer frequency $\left(10^{-5}-10^{-6}\right)$ was observed for transfer of pCC31 from C. coli CC31 into C. jejuni VC83 $\operatorname{Str}^{\mathrm{R}}$. When VC83 containing pTet was used as a donor to transfer pTet into DB179 containing the tagged version of pVir, the transfer frequency was again $10^{-4}$, suggesting that $81-176$ did not restrict incoming DNA from the VC83 donor.

The kinetics of conjugal transfer between C. coli CC31 and C. jejuni VC83 were monitored at various times from 0.5 to $24 \mathrm{~h}$. Peak mating frequency between these strains was observed to take place between 8 and $16 \mathrm{~h}$ of incubation prior to plating on selective media (Fig. 3), although significant transfer was detected as early as $30 \mathrm{~min}$.

A site-specific mutation of the $\mathrm{cmgB} / 4$ gene of pTet in 81176 recA:: aph 3 was constructed as described in Methods. When this strain was used as donor in a cross with VC83 $\mathrm{Str}^{\mathrm{R}}$, no transconjugants were detected, indicating that the $\mathrm{cmgB3} / 4$ gene is required for conjugation proficiency. Since the donor strain carried pVir, it appears that pVir cannot complement the $c m g B 3 / 4$ defect, possibly due to the low overall homology between $\mathrm{cmgB3} / 4$ and the virB4-like gene present in the T4SS of pVir. This indicates that the T4SS

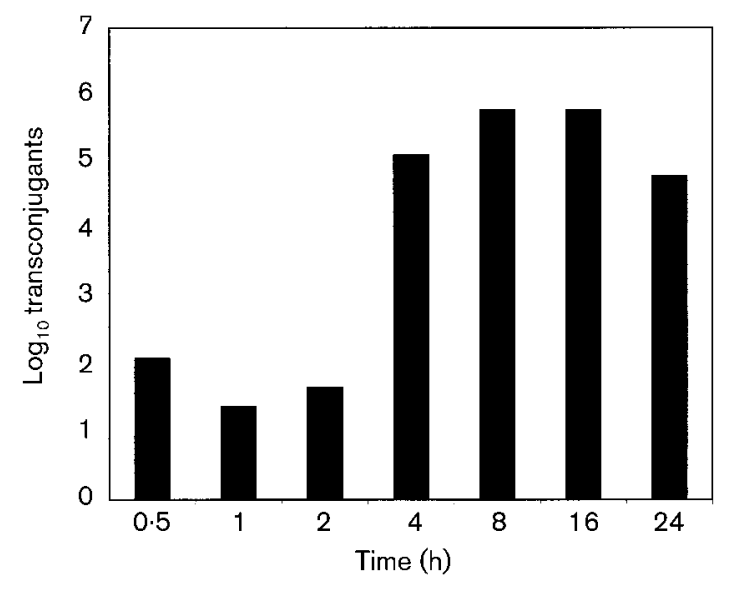

Fig. 3. Kinetics of conjugation between $C$. coli CС31 (pCC31) and C. jejuni VC83. The number of transconjugants represent the number of colonies present on counter-selective media ( $\mathrm{MH}$ agar with tetracycline and streptomycin) per $\mathrm{ml}$ of inoculum divided by the total number of recipient cells per $\mathrm{ml}$. 
carried by pTet and pCC31 is required for conjugation and is functionally distinct from the T4SS carried on pVir.

\section{Comparison to pVir}

Although the two T4SS systems encoded by pVir and pTet in 81-176 share some homology to one another, they appear to serve distinct functions, as mentioned above. Three ORFs on pTet or pCC31 share homology to genes on pVir. One is $c p p 6$, which encodes a predicted protein of $7 \mathrm{kDa}$ with $96 \%$ homology to Cjp19 of pVir, a protein of unknown function. Two ORFs on pTet and pCC31 designated $c p p 7$ and $c p p 51$ share homology to cjp20 on pVir. Analysis of the sequence homology reveals that $c p p 7$ and $c p p 51$ are in fact homologous to the $\mathrm{N}$-terminal and C-terminal sequences of $c j p 20$ from $\mathrm{pVir}$, respectively. This suggests that the Cjp20 homologue in pTet and pCC31 was disrupted through a recombination event.

\section{DISCUSSION}

Plasmids pCC31 $(44.7 \mathrm{~kb})$ and pTet $(45 \cdot 2 \mathrm{~kb})$ are both tetracycline resistance plasmids isolated from clinical isolates of $C$. coli and C. jejuni, respectively. Although these two strains were isolated around 20 years apart and on different continents they showed a remarkable similarity in overall sequence (94.3\% identity) and genomic organization (Fig. 1). Only three genes of unknown function are uniquely found on one of the plasmids, two of which have known homologues in $\mathrm{H}$. pylori.

Apart from the 30 ORFs of unknown function, all of the genes present in pCC31 and pTet are predicted to be involved in plasmid replication and conjugative transfer. The mating pair formation (Mpf) genes involved in conjugation share amino acid similarities to the T4SS of different Brucella species, but have the highest overall homology to the Mpf gene cluster in pVT745 from Actinobacillus actinomycetemcomitans, a periodontal pathogen. The organization of the Mpf gene cluster resembles those of other conjugative plasmids and T4SSs but is most similar to that found in pVT745. In particular, the location of cagT and $\operatorname{TrbM}$ homologues at the end of the T4SS gene cluster is unusual and has only been described previously in pVT745. This strongly suggested that the Mpf gene cluster in these plasmids may have originated from a common ancestor. The similarities in gene organization between pCC31, pTet and pVT745 are not apparent over the rest of the plasmid sequence, although the probable nickase (Cpp17) and a putative lipoprotein of unknown function (Cpp23) also show highest homology to genes found on Actinobacillus plasmids. The replication proteins of pCC31 and pTet showed highest similarity to Rep proteins found in plasmids of the oral spirochaete Treponema denticola (Chauhan \& Kuramitsu, 2004) and Selenomonas ruminantium. The latter is a prominent and functionally diverse species found in the rumen of sheep, cows and goats. Interestingly, repA, the three upstream ORFs of unknown function and the tet( $\mathrm{O})$ gene in pCC31 and pTet have a
$\mathrm{G}+\mathrm{C}$ content that is substantially higher than that of the rest of the plasmid sequence, suggesting that they have a different origin to the rest of the plasmid DNA. Interestingly, DNA sequences of Selenomonas ruminantium submitted to public databases have a similar $\mathrm{G}+\mathrm{C}$ content, but unfortunately, little is known about the genetics of this organism or the function of the various plasmids $(1 \cdot 4-42 \cdot 6 \mathrm{~kb})$ that have been isolated from some strains (Fliegerova et al., 1998). Altogether there are five genes in pCC31 and pTet that have close homologues in the chromosome of $\mathrm{H}$. pylori, one of which is found in the plasticity region. Plasmids found in Helicobacter species have not yet been genetically characterized or sequenced, so it is not known whether any of the ORFs present in pCC31 and pTet have homologues on plasmids found in Helicobacter. This would be interesting, given that these two organisms are closely related, and together with the ruminant bacterium Wolinella succinogenes belong to the epsilon subclass of the proteobacteria. The genes encoding the putative enzymes involved in DNA processing and transfer such as the nickase, helicase, primase, invertase and single-stand-binding protein are all scattered across both plasmids and do not obviously have a common origin. Thus, plasmids pCC31 and pTet are true composites, with a mosaic structure comprising blocks of genes that seem to have been acquired from bacteria that inhabit the oral and intestinal tract of animals. Campylobacter has been identified as a commensal in the gastrointestinal tracts of several species of domestic animals, as well as wildlife species, and is especially abundant in avian species such as chickens, where it can reach up to $10^{10}$ c.f.u. per g caecal contents. The mosaic structure of these plasmids could reflect the recognized potential for gene transfer and recombination in the complex ecosystem of the animal host but the natural competence of Campylobacter for transformation with exogenous DNA may also be a factor contributing to their evolution and mosaic composition.

We have demonstrated that pCC31 and pTet are selfmobilizable and capable of transfer between $C$. jejuni and C. coli strains at frequencies of between $10^{-4}$ and $10^{-6}$, depending on the existence of restriction barriers. The full host-range of these plasmids is not known and difficult to predict as the repA gene exhibits closest homology with genes in plasmids from organisms for which genetic tools have not yet been developed. Preliminary studies with pCC31 and pTet indicated that transfer to E. coli was not possible, as reported previously for $\mathrm{Tet}^{\mathrm{R}}$ plasmids in Campylobacter (Tenover et al., 1985).

Since this paper was first submitted a study was published showing that 16 out of 56 clinical isolates of $C$. jejuni from the area of Göttingen in Germany harbour plasmids varying in size from 6 to $66 \mathrm{~kb}$ (Schmidt-Ott et al., 2004). Only one of these plasmids was a homologue of $\mathrm{pVir}$, the virulence plasmid previously characterized by Bacon et al. (2002). The relatedness of eight plasmids within a subgroup distinct from pVir was established by Southern-blot hybridization 
using a collection of nine PCR-amplified DNA probes from plasmid pCjA13.

Probe $\mathrm{D}$ used in the above study encodes the tet $(\mathrm{O})$ gene, which has $94.7 \%$ identity to that found in pCC 31 . The primer sequences used to amplify probes $\mathrm{B}, \mathrm{D}, \mathrm{F}, \mathrm{H}$ and I in pCjA13 (Schmidt-Ott et al. 2004) were also present in pCC31 (allowing for 1-2 bp mismatches) and were predicted to amplify DNA fragments of similar length, suggesting that these regions are conserved. The sequence of six ORFs present on a $6.8 \mathrm{~kb} B g l \mathrm{II}$ DNA fragment of pCjA13 were submitted to the database and four of these shared closest homology with the virB8, virB9, virB10 and virB11 genes in pVT745 from Actinobacillus actinomycetemcomitans and a high degree of sequence identity to the homologues in pCC31 and pTet (e.g. 89\% amino acid identity between virB9 of pCjA13 and pCC31). However, the last two ORFs of pCjA13 that encoded genes of unknown function were not present in pCC31 or pTet.

In conclusion, it seems that a subgroup of conjugative plasmids with extensive homology to pCC31 and pTet are relatively prevalent in clinical isolates of $C$. jejuni (i.e. eight out of 16 strains harbouring plasmids). It is most likely that the use of tetracycline in poultry has been a contributing factor to the spread of these mobilizable plasmids, but it is also possible that there has been further selection associated with properties conferred by the many uncharacterized ORFs in pCC31 and pTet. The complete sequences of two conjugative plasmids from Campylobacter has provided us with new insights into the evolution of Campylobacter plasmids and the plasticity of the plasmid gene pool. We now intend to investigate the function of the numerous uncharacterized genes encoded in pTet and pCC31 and determine the potential for gene transfer between different bacterial species in the animal ecosystem inhabited by Campylobacter.

\section{ACKNOWLEDGEMENTS}

This work was supported at the Naval Medical Research Centre by the Military Infectious Diseases Research Program and the Office of Naval Research. The work at the Institute of Food Research was supported by a McDonald's Research Grant, BBSRC Core Strategic Grant and a BBSRC studentship. We thank Dlawer Ala'Aldeen, Queen's Medical Centre, Nottingham for his encouragement and supply of C. coli strain CC31. The authors thank Samantha Weavers and Therese Hall for help with preparation of the manuscript.

\section{REFERENCES}

Alm, R. A. \& Trust, T. J. (1999). Analysis of the genetic diversity of Helicobacter pylori: the tale of two genomes. J Mol Med 77, 834-846.

Alm, R. A., Guerry, P. \& Trust, T. J. (1993). Distribution and polymorphism of the flagellin genes from isolates of Campylobacter coli and Campylobacter jejuni. J Bacteriol 175, 3051-3057.

Alm, R. A., Ling, L. S., Moir, D. T. \& 20 other authors (1999). Genomic-sequence comparison of two unrelated isolates of the human gastric pathogen Helicobacter pylori. Nature 397, 176-180.
Alonso, J. C., Weise, F. \& Rojo, F. (1995). The Bacillus subtilis histone-like protein Hbsu is required for DNA resolution and DNA inversion mediated by the $\beta$ recombinase of plasmid pSM19035. J Biol Chem 270, 2938-2945.

Anderson, L. B., Hertzel, A. V. \& Das, A. (1996). Agrobacterium tumefaciens VirB7 and VirB9 form a disulfide-linked protein complex. Proc Natl Acad Sci U S A 93, 8889-8894.

Bacon, D. J., Alm, R. A., Burr, D. H., Hu, L., Kopecko, D. J., Ewing, C. P., Trust, T. J. \& Guerry, P. (2000). Involvement of a plasmid in virulence of Campylobacter jejuni 81-176. Infect Immun 68, 4384-4390.

Bacon, D. J., Alm, R. A., Hu, L., Hickey, T. E., Ewing, C. P., Batchelor, R. A., Trust, T. J. \& Guerry, P. (2002). DNA sequence and mutational analyses of the pVir plasmid of Campylobacter jejuni 81-176. Infect Immun 70, 6242-6250.

Balzer, D., Pansegrau, W. \& Lanka, E. (1994). Essential motifs of relaxase (TraI) and TraG proteins involved in conjugative transfer of plasmid RP4. J Bacteriol 176, 4285-4295.

Beaupre, C. E., Bohne, J., Dale, E. M. \& Binns, A. N. (1997). Interactions between VirB9 and VirB10 membrane proteins involved in movement of DNA from Agrobacterium tumefaciens into plant cells. J Bacteriol 179, 78-89.

Black, R. E., Levine, M. M., Clements, M. L., Hughes, T. P. \& Blaser, M. J. (1988). Experimental Campylobacter jejuni infection in humans. $J$ Infect Dis 157, 472-479.

Bruand, C., Ehrlich, S. D. \& Janniere, L. (1995). Primosome assembly site in Bacillus subtilis. EMBO J 14, 2642-2650.

Cabezon, E., Lanka, E. \& de la Cruz, F. (1994). Requirements for mobilization of plasmids RSF1010 and ColE1 by the IncW plasmid R388: trwB and RP4 traG are interchangeable. J Bacteriol 176, 4455-4458.

Cao, T. B. \& Saier, M. H., Jr (2001). Conjugal type IV macromolecular transfer systems of Gram-negative bacteria: organismal distribution, structural constraints and evolutionary conclusions. Microbiology 147, 3201-3214.

Censini, S., Lange, C., Xiang, Z., Crabtree, J. E., Ghiara, P., Borodovsky, M., Rappuoli, R. \& Covacci, A. (1996). cag, a pathogenicity island of Helicobacter pylori, encodes type I-specific and disease-associated virulence factors. Proc Natl Acad Sci U S A 93, 14648-14653.

Chauhan, S. \& Kuramitsu, H. K. (2004). Sequence analysis of plasmid pTS1 isolated from oral spirochetes. Plasmid 51, 61-65.

Christie, P. J. \& Vogel, J. P. (2000). Bacterial type IV secretion: conjugation systems adapted to deliver effector molecules to host cells. Trends Microbiol 8, 354-360.

Christie, P. J., Ward, J. E., Winans, S. C. \& Nester, E. W. (1988). The Agrobacterium tumefaciens virE2 gene product is a single-strandedDNA-binding protein that associates with T-DNA. J Bacteriol 170, 2659-2667.

Covacci, A., Telford, J. L., Del Giudice, G., Parsonnet, J. \& Rappuoli, R. (1999). Helicobacter pylori virulence and genetic geography. Science 284, 1328-1333.

Eisenbrandt, R., Kalkum, M., Lurz, R. \& Lanka, E. (2000). Maturation of IncP pilin precursors resembles the catalytic Dyad-like mechanism of leader peptidases. J Bacteriol 182, 6751-6761.

Fliegerova, K., Benada, O. \& Flint, H. J. (1998). Large plasmids in ruminal strains of Selenomonas ruminantium. Lett Appl Microbiol 26, 243-247.

Galli, D. M., Chen, J., Novak, K. F. \& Leblanc, D. J. (2001). Nucleotide sequence and analysis of conjugative plasmid pVT745. J Bacteriol $183,1585-1594$. 
Gaynor, E. C., Ghori, N. \& Falkow, S. (2001). Bile-induced 'pili' in Campylobacter jejuni are bacteria-independent artifacts of the culture medium. Mol Microbiol 39, 1546-1549.

Guerry, P., Pope, P. M., Burr, D. H., Leifer, J., Joseph, S. W. \& Bourgeois, A. L. (1994). Development and characterization of recA mutants of Campylobacter jejuni for inclusion in attenuated vaccines. Infect Immun 62, 426-432.

Guerry, P., Ewing, C. P., Hickey, T. E., Prendergast, M. M. \& Moran, A. P. (2000). Sialylation of lipooligosaccharide cores affects immunogenicity and serum resistance of Campylobacter jejuni. Infect Immun 68, 6656-6662.

Janniere, L., Gruss, A. \& Ehrlich, S. D. (1993). Plasmids, Bacillus subtilis and other gram-positive bacteria. In Plasmids, pp. 625-644. Edited by A. L. Sonenshein, J. A. Hoch \& R. Losick. Washington, DC: American Society for Microbiology.

Kersulyte, D., Velapatino, B., Mukhopadhyay, A. K., Cahuayme, L., Bussalleu, A., Combe, J., Gilman, R. H. \& Berg, D. E. (2003). Cluster of type IV secretion genes in Helicobacter pylori's plasticity zone. J Bacteriol 185, 3764-3772.

Konieczny, I. (2003). Strategies for helicase recruitment and loading in bacteria. EMBO Rep 4, 37-41.

Korlath, J. A., Osterholm, M. T., Judy, L. A., Forfang, J. C. \& Robinson, R. A. (1985). A point-source outbreak of campylobacteriosis associated with consumption of raw milk. J Infect Dis 152, 592-596.

Krause, S., Barcena, M., Pansegrau, W., Lurz, R., Carazo, J. M. \& Lanka, E. (2000). Sequence-related protein export NTPases encoded by the conjugative transfer region of RP4 and by the cag pathogenicity island of Helicobacter pylori share similar hexameric ring structures. Proc Natl Acad Sci U S A 97, 3067-3072.

Kuipers, E. J., Israel, D. A., Kusters, J. G. \& Blaser, M. J. (1998). Evidence for a conjugation-like mechanism of DNA transfer in Helicobacter pylori. J Bacteriol 180, 2901-2905.

Lee, C. Y., Tai, C. L., Lin, S. C. \& Chen, Y. T. (1994). Occurrence of plasmids and tetracycline resistance among Campylobacter jejuni and Campylobacter coli isolated from whole market chickens and clinical samples. Int J Food Microbiol 24, 161-170.

Lessl, M., Balzer, D., Pansegrau, W. \& Lanka, E. (1992). Sequence similarities between the RP4 Tra2 and the Ti VirB region strongly support the conjugation model for T-DNA transfer. J Biol Chem 267, 20471-20480.

Moncalian, G., Cabezon, E., Alkorta, I., Valle, M., Moro, F., Valpuesta, J. M., Goni, F. M. \& de la Cruz, F. (1999). Characterization of ATP and DNA binding activities of TrwB, the coupling protein essential in plasmid R388 conjugation. J Biol Chem 274, 36117-36124.

Nachamkin, I., Allos, B. M. \& Ho, T. (1998). Campylobacter species and Guillain-Barré syndrome. Clin Microbiol Rev 11, 555-567.

Oberhelman, R. A. \& Taylor, D. E. (2000). Campylobacter infections in developing countries. In Campylobacter, pp. 139-153. Edited by I. Nachamkin \& M. J. Blaser. Washington, DC: American Society for Microbiology.

Pansegrau, W. \& Lanka, E. (1996). Enzymology of DNA transfer by conjugative mechanisms. Prog Nucleic Acid Res Mol Biol 54, 197-251.

Parkhill, J., Wren, B. W., Mungall, K. \& 18 other authors (2000). The genome sequence of the food-borne pathogen Campylobacter jejuni reveals hypervariable sequences. Nature 403, 665-668.

Schmidt-Eisenlohr, H., Domke, N., Angerer, C., Wanner, G., Zambryski, P. C. \& Baron, C. (1999). Vir proteins stabilize VirB5 and mediate its association with the $\mathrm{T}$ pilus of Agrobacterium tumefaciens. J Bacteriol 181, 7485-7492.
Schmidt-Ott, R., Pohl, S., Burghard, S., Weig, M. \& Groß, U. (2004). Identification and characterization of a major subgroup of conjugative Campylobacter jejuni plasmids. J Infect (in press).

Schneiker, S., Keller, M., Droge, M., Lanka, E., Puhler, A. \& Selbitschka, W. (2001). The genetic organization and evolution of the broad host range mercury resistance plasmid pSB102 isolated from a microbial population residing in the rhizosphere of alfalfa. Nucleic Acids Res 29, 5169-5181.

Spudich, G. M., Fernandez, D., Zhou, X. R. \& Christie, P. J. (1996). Intermolecular disulfide bonds stabilize VirB7 homodimers and VirB7/VirB9 heterodimers during biogenesis of the Agrobacterium tumefaciens T-complex transport apparatus. Proc Natl Acad Sci U S A 93, 7512-7517.

Strack, B., Lessl, M., Calendar, R. \& Lanka, E. (1992). A common sequence motif, -E-G-Y-A-T-A-, identified within the primase domains of plasmid-encoded I- and P-type DNA primases and the $\alpha$ protein of the Escherichia coli satellite phage P4. J Biol Chem 267, 13062-13072.

Takai, S., Hines, S. A., Sekizaki, T. \& 10 other authors (2000). DNA sequence and comparison of virulence plasmids from Rhodococcus equi ATCC 33701 and 103. Infect Immun 68, 6840-6847.

Tauch, A., Schneiker, S., Selbitschka, W. \& 13 other authors (2002). The complete nucleotide sequence and environmental distribution of the cryptic, conjugative, broad-host-range plasmid pIPO2 isolated from bacteria of the wheat rhizosphere. Microbiology 148, 1637-1653.

Taylor, D. E. (1986). Plasmid-mediated tetracycline resistance in Campylobacter jejuni: expression in Escherichia coli and identification of homology with streptococcal class M determinant. J Bacteriol 165, 1037-1039.

Taylor, D. E., De Grandis, S. A., Karmali, M. A. \& Fleming, P. C. (1981). Transmissible plasmids from Campylobacter jejuni. Antimicrob Agents Chemother 19, 831-835.

Taylor, D. E., Chang, N., Garner, R. S., Sherburne, R. \& Mueller, L. (1986). Incidence of antibiotic resistance and characterization of plasmids in Campylobacter jejuni strains isolated from clinical sources in Alberta, Canada. Can J Microbiol 32, 28-32.

Tenover, F. C., Williams, S., Gordon, K. P., Nolan, C. \& Plorde, J. J. (1985). Survey of plasmids and resistance factors in Campylobacter jejuni and Campylobacter coli. Antimicrob Agents Chemother 27, 37-41.

Thorstenson, Y. R., Kuldau, G. A. \& Zambryski, P. C. (1993). Subcellular localization of seven VirB proteins of Agrobacterium tumefaciens: implications for the formation of a T-DNA transport structure. J Bacteriol 175, 5233-5241.

Tomb, J. F., White, O., Kerlavage, A. R. \& 39 other authors (1997). The complete genome sequence of the gastric pathogen Helicobacter pylori. Nature 388, 539-547.

Vergunst, A. C., Schrammeijer, B., den Dulk-Ras, A., de Vlaam, C. M., Regensburg-Tuink, T. J. \& Hooykaas, P. J. (2000). VirB/D4dependent protein translocation from Agrobacterium into plant cells. Science 290, 979-982.

Wassenaar, T. M. \& Blaser, M. J. (1999). Pathophysiology of Campylobacter jejuni infections of humans. Microbes Infect $\mathbf{1}$, 1023-1033.

Yao, R., Alm, R. A., Trust, T. J. \& Guerry, P. (1993). Construction of new Campylobacter cloning vectors and a new mutational cat cassette. Gene 130, 127-130.

Zechner, E. L., de la Cruz, F., Eisenbrandt, R. \& 8 other authors (2000). Conjugative-DNA transfer processes. In The Horizontal Gene Pool - Bacterial Plasmids and Gene Spread, pp. 87-174. Edited by C. M. Thomas. Amsterdam: Harwood Academic. 